\title{
Porous materials show superhydrophobic to superhydrophilic switching
}

\author{
Neil J. Shirtcliffe,* Glen McHale, Michael I. Newton, Carole C. Perry and Paul Roach
}

\author{
*School of Biomedical and Natural Sciences, Nottingham Trent University, Clifton Lane Nottingham, NG11 8NS, UK. Fax:+44 \\ (0)115 8483384; Tel:+44 (0)115 8486375; E-mail: neil.shirtcliffe@ntu.ac.uk
}

Postprint of original article: N.J. Shirtcliffe, G. McHale, M.I. Newton, C.C. Perry and P. Roach, Porous materials show superhydrophobic to superhydrophilic switching, Chem. Comm. (25) (2005) 3135-3137. (See also Nature Highlight/News "Quick change for super sponge" Published on-line 20/7/05). (Front cover image). DOI: 10.1039/b502896e

\section{Switching between superhydrophobicity and superhydrophilicity in porous materials was predicted theoretically and demonstrated experimentally with the example of thermally induced contact angle change; tunability of this system was also demonstrated.}

A surface that is both hydrophobic and rough can become superhydrophobic; the roughness magnifies the effect of the hydrophobicity and can lead to surfaces being so hydrophobic that water drops on them appear to be spherical. The same effect can be observed with other liquids on the surface of rough solids. If the roughness is slight liquid can follow the contours of the surface, but if it is very high water tends to bridge the tops of peaks of the roughness. Both cases can lead to increases in contact angle for small droplets of liquid deposited on the surface. ${ }^{1-4}$ The same principle applies for liquids other than water, but the transition is then a super-non-wetting to super-wetting one. This effect has been observed on natural and synthetic surfaces. ${ }^{5,6}$

Porous materials will either show bridging type non-wetting or will fill with liquid. When liquid sits on a flat, heterogeneous surface or bridges the peaks of a rough one, the Cassie-Baxter model, (Eqn. 1) ${ }^{7}$ applies; the liquid drop suspends itself across surface protrusions and the contact angle can be derived from the sum of the cosines of the angles on each type of interface multiplied by their fractional area.

$$
\cos \theta_{r}=f \cos \theta_{s}+(1-f) \cos \theta_{x}
$$

In eqn. $1, \theta_{r}$ is the contact angle of the liquid on the rough surface, $\theta_{s}$ the contact angle of the liquid on a smooth surface having the same chemistry as the rough surface, $f$ is the fraction of the base of the drop in contact with the solid and (1-f) is the remaining fraction of the drop base. $\theta_{x}$ is the contact angle of a liquid on air in the case of suspended drops; this is $180^{\circ}$. The formula can be used for any heterogenous surface with a flat water interface. Significantly on a porous surface filled with a liquid $\theta_{x}$ would become $0^{\circ}$ for droplets of the same liquid added to the surface and these droplets would then tend to spread more than on a flat surface of the solid material. This difference in the meaning of $\theta_{x}$, corresponding to whether gaps between surface protrusions are empty or filled changes $\cos \theta_{x}$ from -1 to 1 , allowing Eqn. 1 to be reduced to Eqn. 2.

$$
\cos \theta_{r}=f \cos \theta_{s} \pm(1-f)
$$

Eqn. 2 applies for porous materials where the pores can either be empty, in which case the negative sign would be used, or filled with the same liquid as is present on the surface, when the positive sign would be used. If the material has a high volume fraction of pores, $f$ will be small so the second component of the equation will dominate and one would expect liquid to switch from very high (nearly $180^{\circ}$ ) to very low (nearly $0^{\circ}$ ) contact angles when the contact angle on a flat surface is varied by a small amount around the value of $90^{\circ}$; at this point sudden intrusion into the pores will occur. This is likely to be particularly effective on materials with interconnected pores as the liquid in the material will fill pores ahead of the advancing drop.

Enhancement of contact angle variation using roughness has been suggested as a measurement technique in previous work $^{8,9}$, but mesoporous materials may have even sharper transitions than rough surfaces as the pores can only be empty or filled, partial states are not energetically favored.

The porous materials used in this report were produced using a phase separation method in which a hardening process freezes a phase separation that occurs concurrently with hardening of one of the phases. $^{10}$ This method produces co-continuous materials consisting of a solid phase and a liquid phase. When the liquid was removed a porous structure remained. The system used was the condensation of an organo-triethoxysilane in a mixture of organic solvent and water. Sol-gel materials, such as this, show promise as superhydrophobic surfaces, ${ }^{11,12}$ particularly as hard, superhydrophobic coatings but also as bulk materials ${ }^{13}$ and are relatively cheap to produce. ${ }^{14}$ Reaction occurs through hydrolysis of the ethoxy groups and polymerisation of the silanol groups thus formed. Polymerisation causes a decrease in dipole moment, leading to hydrophobic phase separation. The dried material presents the organic group on its surface, causing the foams to be superhydrophobic. The structure of these materials has been reported in an earlier paper ${ }^{15}$ where we reported that the advancing contact angles on such gels heated to $300^{\circ} \mathrm{C}$ were $155 \pm 2^{\circ}$. If a flat surface of the organosilica is heated it becomes gradually less hydrophobic, but the foams undergo a more sudden change. The contact angle values are similar to those achieved by other researchers using similar materials. ${ }^{16-18}$

The central image in Fig. 1 is an electron micrograph of a typical material used showing the large pore fraction (measured previously as $75 \%$ ) that generates the Cassie-Baxter type superhydrophobicity of these materials. The structure of the material did not significantly change on heating to $400{ }^{\circ} \mathrm{C}$.

The top row of Fig. 1 shows two samples of methyltriethoxysilane (MTEOS) sol-gel foams in which a drop of water containing dye sat on the top of the sample heated to $390^{\circ} \mathrm{C}$ 


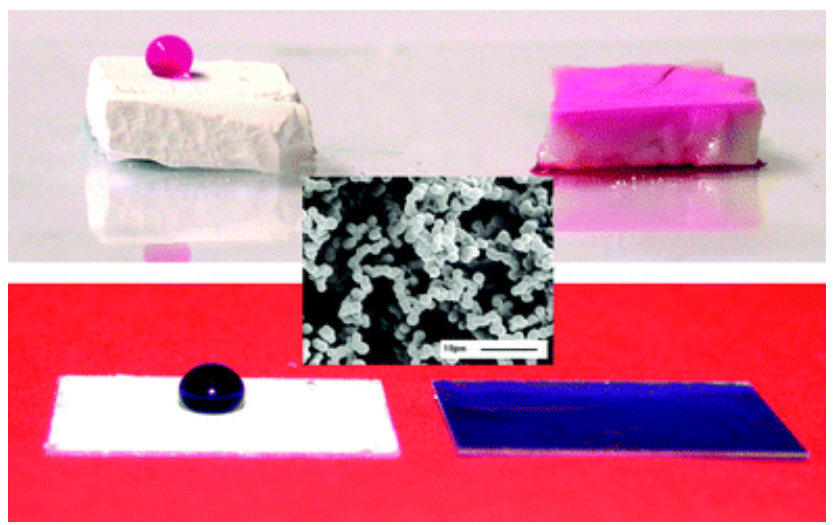

Fig. 1 Top, phenolphthalein in water on MTEOS sol-gel foams heated to $390{ }^{\circ} \mathrm{C}$ (left) and $400{ }^{\circ} \mathrm{C}$ (right). Centre, SEM of sol-gel foam unheated. Bottom, foam films on glass cover slips with (left) drop of water with brilliant blue $\mathrm{G}$ and (right) imbibed.

but was fully absorbed into that heated to $400^{\circ} \mathrm{C}$. This transition was effectively binary, either the drop penetrated or it did not. Varying the temperature of this transition will be considered later in this communication. A practical device could consist of a line of thin gel films with different transition temperatures on a baseplate that would be dipped into a coloured solution after heating. Hydrophilic gels would absorb the dye and become coloured while those not reaching their transition temperature would not.

Similar foam materials were prepared as adherent films, which would be more practical as a device. Examples of these are shown in the bottom row of Fig. 1 with a drop of dyed water on a hydrophobic and a hydrophilic foam film.

To investigate the mechanism behind the change in wettability of the material, infrared spectra were taken after heating gels to various temperatures.

The transmission infrared spectra shown in Fig. 2a were measured using a single $\mathrm{KBr}$ plate containing MTEOS foam. It was heated to different temperatures and infrared spectra measured. The spectra show the C-H stretching region, which is almost unchanged upon heating up to $400^{\circ} \mathrm{C}$, apart from a slight change in peak shape at around $2925 \mathrm{~cm}^{-1}$ between 200 and $240^{\circ} \mathrm{C}$. This indicates that methyl functionality was not lost during the hydrophobic to hydrophilic transition, which occurred at just below $400^{\circ} \mathrm{C}$ for these samples.

The same region of diffuse reflection spectra (measuring the surface region) of pure samples heated to similar temperatures are shown in Fig. 2 parts b and c. Part b shows spectra taken after heating to 180 and $220{ }^{\circ} \mathrm{C}$, bands at 2930 and $2850 \mathrm{~cm}^{-1}$ corresponding to a $\mathrm{CH}_{2}$ stretch disappear on heating to $220^{\circ} \mathrm{C}$ and a broad band appears at $2810 \mathrm{~cm}^{-1}$. The two $\mathrm{CH}_{2}$ bands probably arise from unreacted ethoxysilyl groups. On heating these would be expected to crosslink with hydroxy groups at low temperature releasing ethanol, which explains the loss of these signals by 220 ${ }^{\circ} \mathrm{C}$. Above this temperature the only $\mathrm{C}-\mathrm{H}$ functionality expected and observed is $\mathrm{Si}-\mathrm{CH}_{3}$. Fig. 2c shows reflectance spectra taken upon further heating. The $\mathrm{CH}_{3}$ bands broaden, possibly due to changes in chemical environment and they lose some intensity. The band at $2810 \mathrm{~cm}^{-1}$, possibly also $\mathrm{CH}_{3}$ groups in a strained environment, did not appear to change once formed.
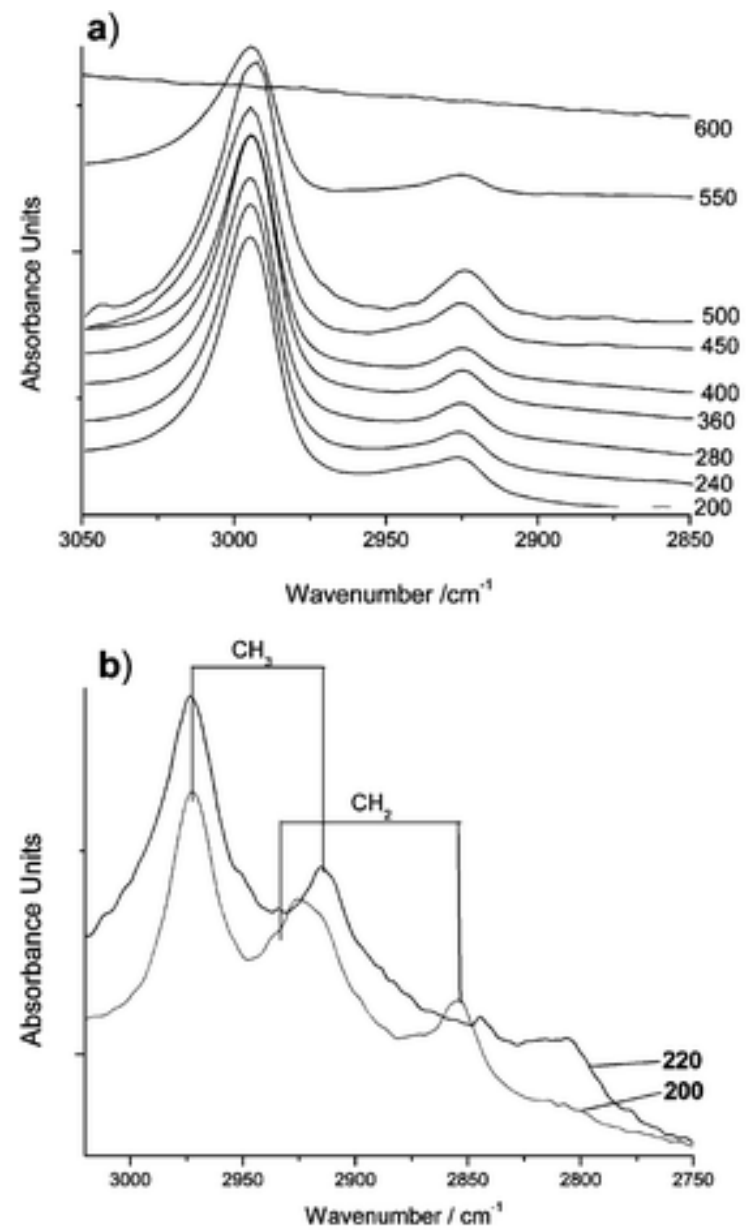

c)

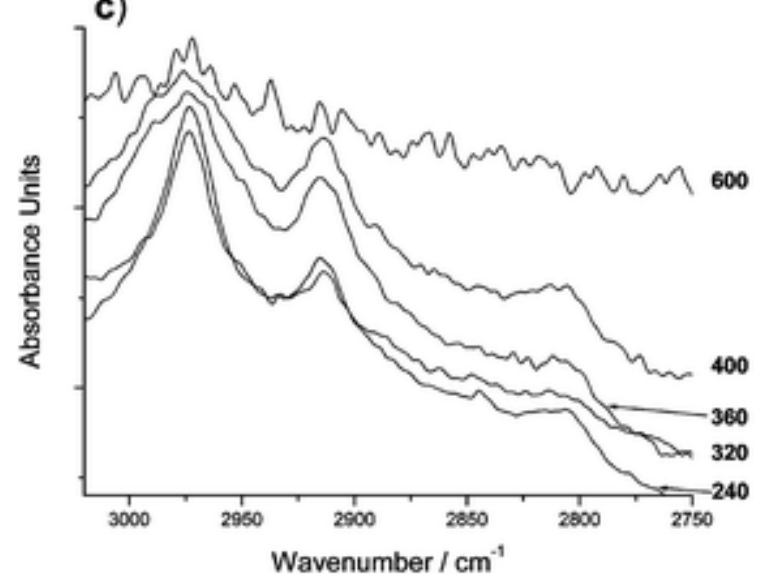

Fig. 2 IR spectra of MTEOS sol-gel materials heated to various temperatures; a) transmission spectra, b) and c) diffuse reflection spectra.

The infrared data suggest that the surface of the gel is initially covered with methyl and ethoxy groups. The ethoxy groups are lost at a low temperature long before the materials become hydrophilic. Slight broadening and loss of intensity was observed in $\mathrm{C}-\mathrm{H}$ bands at the surface of the material where bulk measurements showed no changes. Changes in the shape of the $\mathrm{Si}-\mathrm{O}$ region indicate crosslinking of the silica and relative loss of $\mathrm{Si}-\mathrm{C}$ intensity in 
Postprint Version: The following article appeared in Chemical Communications and may be found at http://www.rsc.org/Publishing/Journals/CC/article.asp?doi=b502896e. Copyright C2005 Royal Society of Chemistry

Table 1 Superhydrophobic-hydrophilic transition temperatures

\begin{tabular}{|c|c|}
\hline Gel Composition & Transition $/{ }^{\circ} \mathrm{C}\left( \pm 10^{\circ} \mathrm{C}\right)$ \\
\hline MTEOS & 400 \\
\hline PhTEOS:TEOS $1: 2$ & 275 \\
\hline PhTEOS:TEOS $1: 1$ & 550 \\
\hline PhTEOS:TEOS $2: 1$ & 550 \\
\hline
\end{tabular}

reflection IR backs up the $\mathrm{C}-\mathrm{H}$ band data . To become hydrophilic the surface must become more polar. This could occur by the formation of new groups or by a change in the relative abundances of apolar methyl groups and polar silica species. Methyl groups could be oxidatively cleaved or become hidden in the bulk of the material. The infrared data only show loss of methyl groups from the surface of the material, not where they go. Transmission infrared measurements show that bulk methyl concentration does not change until the temperature exceeds $550{ }^{\circ} \mathrm{C}$. No C-O functionality appeared at any time in the spectra, so there is no evidence that methyl groups are being oxidised. ${ }^{\dagger}$

In order for the transition of the materials from superhydrophobic to superhydrophilic to be of use in a sensor it must be tuneable. If the transition temperature is controlled by the crosslinking of silica rotating organo-groups away from the surface, it should be affected by the density of silicon-organic bonds and the bulk of the organic groups; equally if it is due to the oxidative loss of the organic groups it should be affected by the reactivity of these groups. Sol-gel foams were prepared using varying proportions of phenyl triethoxysilane (PhTEOS) and TEOS to test this. $\mathrm{Ph}-\mathrm{Si}$ is known to be more resistant to heating than $\mathrm{CH}_{3}-\mathrm{Si}^{12}$ and the phenyl group is also bulkier so an increase in switching temperature would be expected.

The temperatures at which switching occurred were increased when larger fractions of PhTEOS were used but decreased considerably when larger fractions of TEOS were used (Table 1). Similarly Rao and Haranath ${ }^{17}$ used MTEOS/TEOS mixtures and observed hydrophilicity at $280{ }^{\circ} \mathrm{C}$. The transition temperatures of PhTEOS and MTEOS containing foams both decreased significantly on increasing the fraction of TEOS. This suggests that the hydrophilic-hydrophobic transition is due to crosslinking of the silica backbone causing redistribution of the organic groups from the surface into the bulk of the material. It is not surprising that the maximum temperature appears to be limited to $550-600{ }^{\circ} \mathrm{C}$ as this is where loss of the $\mathrm{C}-\mathrm{H}$ groups became apparent in the infrared measurements.

A range of foam materials were prepared as bulk and films. Heating caused a gradual increase in hydrophilicity of a flat surface, but a sudden change from superhydrophobicity to absorption in porous ones. This behaviour is described by a modification of Cassie and Baxter's formula where the pores of a porous surface can either be empty or they can be full of the wetting liquid. This situation would result in switching from very high to very low contact angle without intermediate states being

\footnotetext{
${ }^{\dagger}$ Electronic supplementary information (ESI) available: IR transmission and reflection spectra. See

http://www.rsc.org/suppdata/cc/b5/b502896e/
}

possible. The transition from superhydrophobicity to hydrophilicity of these materials shows promise for detecting thermal history with the wetting, or measurement stage taking place at room temperature. Addition of dyes to the liquid or the foam allowed visualisation of the changes. ${ }^{\ddagger}$ Alternatively shorting of electrodes under the foam could be used to detect imbibition events.

Neil J. Shirtcliffe,* Glen McHale, Michael I. Newton, Carole C. Perry, and Paul Roach

${ }^{a}$ School of Biomedical and Natural Sciences, Nottingham Trent University, Clifton Lane Nottingham, NG11 8NS, GB. Fax:+44 (0)115 8483384; Tel:+44 (0)115 8486375; E-mail: neil.shirtcliffe@ntu.ac.uk

\section{Notes and references}

Sol-gel foams were prepared as described in previous work ${ }^{7}$, except that dimethyl formamide (DMF, Acros 99\%) was used as the co-solvent. DMF does not evaporate very rapidly under normal conditions, so solvent exchange was necessary, achieved by placing the samples into a large volume of methanol (Fisher 99\%) and exchanging it each day for three days. Once dry the samples were placed in a Pyrex ${ }^{\circledR}$ beaker and heated to various temperatures to cross-link and eventually oxidise the materials.

Phenyl terminated foams were prepared by mixing defined molar ratios of phenyl triethoxysilane (PhTEOS, Lancaster 97\%) and tetraethoxysilane (TEOS, Aldrich 98\%), ratios used were 2:1, 1:1 and 1:2. DMF was used as co-solvent here and the molar amount of silicon was kept the same as before so as to maintain the molar ratios of silicon, water and acid.

Infrared measurements were taken using a Nicolet Magna IR-750 with a Spectra-Tech diffuse reflection accessory and $\mathrm{KBr}$ plateholder. $\mathrm{KBr}$ plates were prepared using $2 \mathrm{mg}$ of sol-gel foam in $200 \mathrm{mg}$ of $\mathrm{KBr}$.

1 A. Adamson, Physical Chemistry of Surfaces, 5th Edn., Wiley: New York, 1990.

2 A. Otten and S. Herminghaus, Langmuir, 2004, 20 (6), 2405-2408.

3 P. De Gennes, Rev. Mod. Phys., 1985, 57, 827-863.

4 R. Wenzel, Ind. Eng. Chem., 1936, 28, 988.

5 W. Barthlott and C. Neinhuis, Planta, 1997, 202 (1), 1-8.

6 S. Shibuichi, T. Yamamoto, T. Onda and K. Tsujii, J. Phys. Chem., 1996, 100, 19512-19517.

7 D. Quéré, Physica A, 2002, 313 (1-2), 32-46.

8 G. McHale, N. Shirtcliffe and M. Newton, Analyst, 2004, 129 (4), 284 287.

9 R. Rosario, D. Gust, A. Garcia, M. Hayes, J. Taraci, T. Clement, J. Dailey and S. Picraux, J. Phys. Chem. B, 2004, 108 (34), 1264012642.

10 R. Takahashi, K. Nakanishi and N. Soga, J. Ceram. Soc. Jpn., 1998, 106 (8), 772-777.

11 K. Tadanaga, K. Kitamuro, A. Matsuda and T. Minami, J. Sol-Gel Sci. Technol., 2003, 26 (1-3), 705-708.

12 K. Tadanaga, J. Morinaga and T. Minami, J. Sol-Gel Sci. Technol., 2000, 19, 211-214.

13 A. Rao and M. Kulkarni, Mater. Res. Bull., 2002, 37 (9), 1667-1677.

\footnotetext{
${ }^{+}$Sol-gel foams were prepared as described in previous work, ${ }^{7}$ except that dimethyl formamide (DMF, Acros 99\%) was used as the co-solvent. DMF does not evaporate very rapidly under normal conditions, so solvent exchange was necessary, achieved by placing the samples into a large volume of methanol (Fisher 99\%) and exchanging it each day for three days. Once dry the samples were placed in a Pyrex® beaker and heated to various temperatures to cross-link and eventually oxidise the materials.Phenyl terminated foams were prepared by mixing defined molar ratios of phenyl triethoxysilane (PhTEOS, Lancaster 97\%) and tetraethoxysilane (TEOS, Aldrich 98\%), ratios used were 2 1, 11 and 12. DMF was used as co-solvent here and the molar amount of silicon was kept the same as before so as to maintain the molar ratios of silicon, water and acid.Infrared measurements were taken using a Nicolet Magna IR 750 with a Spectra-Tech diffuse reflection accessory and $\mathrm{KBr}$ plateholder. $\mathrm{KBr}$ plates were prepared using $2 \mathrm{mg}$ of sol-gel foam in $200 \mathrm{mg}$ of $\mathrm{KBr}$.
} 
Postprint Version: The following article appeared in Chemical Communications and may be found at http://www.rsc.org/Publishing/Journals/CC/article.asp?doi=b502896e. Copyright C2005 Royal Society of Chemistry Communication

14 L. Klein (ed.), Sol-Gel Technology For Thin Films, Fibers, Preforms, Electronics and Speciality Shapes, Noyes: New Jersey, 1998.

15 N. Shirtcliffe, G. McHale, M. Newton and C. Perry, Langmuir, 2003, 19 (14), 5626-5631.

16 H. Erbil, A. Demirel, Y. Avci and O. Mert, Science, 2003, 299 5611), 1377-1380.

17 A. Rao and D. Haranath, Microporous Mesoporous Mater., 1999, 30 (2-3), 267-273.

18 A. Roig, E. Mollins, S. Martinez, M. Moreno-Manas and A. Vallribera, Chem. Commun., 2004, 20, 2316-2317.

19 M. Brook, Silicon in organic, organometallic and polymer chemistry, Wiley: New York, 2000. 